\title{
Los videojuegos, herramientas para el cambio. Un estudio desde la teoría de la actividad
}

\author{
Laura Méndez y Pilar Lacasa ${ }^{2}$
}

1 Departamento de Psicología Evolutiva y de la Educación, Universidad Nacional de Educación a Distancia (UNED) Madrid.

${ }^{2}$ Departamento de Comunicación, Universidad de Alcalá, Madrid

\section{España}

Correspondencia: Laura Méndez Zaballos. UNED. Facultad de Psicologia. C/ Juan del Rosal, nº 10, 28040. Madrid. España. E-mail: $\underline{\text { lmendez@psi.uned.es }}$

(C) Education y Psychology I+D+i and Ilustre Colegio Oficial de la Psicología de Andalucía Oriental (Spain) 


\section{Resumen}

Introducción. El propósito de este trabajo es ofrecer un marco de análisis para interpretar, desde la perspectiva de los participantes, las trasformaciones que ocurren en un aula cuando utilizamos videojuegos comerciales. Mostraremos cómo la teoría de la actividad (AT) permite explicar e interpretar estos cambios.

Método. Se presenta un estudio de casos, desde un enfoque cualitativo y etnográfico, en el que exploramos las trasformaciones que tienen lugar en un aula de apoyo de un instituto de secundaria, cuando introducimos el videojuego Sim3. Los participantes son la profesora de apoyo y dos grupos de estudiantes con necesidades educativas especiales (12-16 años).

Resultados. El videojuego, como instrumento mediador, introduce en el aula prácticas, códigos y lenguajes que provocan tensiones en los elementos del sistema (el objeto de la actividad, la relación y papel de los participantes en la comunidad, los resultados de la actividad y las reglas que las organizan). La superación de estas tensiones ha supuesto la trasformación del aula de apoyo en un sistema nuevo y más facilitador para dar respuesta a las necesidades educativas especiales de los estudiantes.

Discusión. Este estudio presenta un marco para el análisis, que partiendo de la teoría de la actividad, ofrece algunas claves para interpretar el papel de instrumentos tecnológicos, como el videojuego, en la escuela: la importancia de trascender el aula para fijarnos en otros sistemas con los que está vinculada, la necesidad de contemplar el videojuego como objeto cultural, a través del que se introducen nuevas prácticas que entran en contradicción con las que, profesores y estudiantes, llevan a cabo en las aulas tradicionales, y finalmente, la importancia de centrar el análisis en las múltiples perspectivas y "voces" de los participantes y en cómo sus interpretaciones, creencias y concepciones, están en la base del cambio.

Palabras Clave: videojuegos, teoría de la actividad, cambio educativo, educación especial 


\title{
Videogames, tools for change: a study based on activity theory
}

\begin{abstract}
Introduction. The purpose of this paper is to provide a framework of analysis to interpret, from the perspective of the participants, the transformations that occur in a classroom when we use commercial video games. We will show how the theory of activity (AT) allows to explain and interpret these changes.
\end{abstract}

Method. We present a study of cases, from a qualitative and ethnographic approach, in which we explore the transformations that take place in a classroom in support of a secondary school, when we introduce the Sim3 videogame. The participants are the teacher's support and two groups of students with special educational needs (12-16 years).

Results. Videogames, as instrument mediator, enters the classroom practices, co-codes and languages causing tensions in the elements of the system (the object of the activity, the relationship and role of participants in the community, the results of the activity and the rules that organize them). The overcoming of these tensions has been the transformation of classroom support in a new system and more facilitator to respond to the special educational needs of the students.

Discussion. This study presents a framework for analysis, which, on the basis of the theory of activity, offers some keys to interpret the role of instruments technology-magical, like the video game, in the school: the importance of going beyond the classroom to look at other systems with which it is linked, the need to consider videogames as a cultural object, which introduced new practices that come into contradiction with the teachers and students, carried out in the traditional classroom, and finally, the importance of focusing the analysis in multiple perspectives and 'voices' of the participants and their interpretations, beliefs and conceptions, are at the base of the change.

Keywords: video games, theory of activity, educational change, special education.

Reception: 09.14.15 Initial acceptance: 10.15.15 Final acceptance: 06.21.15 


\section{Introducción}

El propósito de este trabajo es ofrecer un marco de análisis para interpretar, desde la perspectiva de los participantes, las trasformaciones que ocurren en un aula cuando utilizamos videojuegos comerciales como recurso educativo. Mostraremos cómo la teoría de la actividad (AT) permite explicar e interpretar estos cambios. A partir de este marco conceptual consideramos la actividad que desarrollan estudiantes y profesores como un sistema orientado hacia un objeto colectivo, culturalmente mediado y caracterizado por contradicciones (o tensiones sistémicas), que son un camino para el desarrollo y conducen a la innovación y al cambio (Engestrom, 2001). Este modelo teórico ofrece una herramienta conceptual muy interesante para analizar escenarios donde conviven instrumentos tradicionales con nuevos artefactos tecnológicos y nos permite abordar el proceso de transformación provocado por las tensiones surgidas al interaccionar elementos de sistemas que comparten el mismo objeto, por ejemplo, el aula tradicional y un aula donde se ha introducido un videojuego, instrumento propio de un contexto no formal.

Tomando como referencia este modelo y trabajos realizados en los últimos años (Fujioka, 2014; Joy y Murphy, 2012; Larripa y Euasquin 2010), el objetivo de este artículo es analizar las tensiones surgidas al introducir una práctica típica del ocio juvenil, un videojuego, en un contexto formal y cómo estas tensiones han trasformado los diferentes elementos del aula, entendida como sistema de actividad: el propósito de la actividad, las metas de los participantes, la distribución de papeles entre ellos, las reglas y normas que definen la actividad y el papel de los instrumentos mediadores, en especial del videojuego.

\section{La teoría de la actividad, un modelo para el análisis}

La idea de la tecnología como vehículo para trasformar el proceso de enseñanzaaprendizaje, ha estado en la base de anteriores trabajos que hemos realizado (Lacasa, Méndez, y Martínez, 2008; Monjelat y Méndez, 2012). En ellos pudimos comprobar cómo la tecnología, en concreto los videojuegos, además de posibilitar el desarrollo de habilidades, se convertía en un elemento trasformador de los escenarios educativos, incluidos aquellos pensados para atender a las necesidades de estudiantes con necesidades educativas especiales. Este descubrimiento nos planteó varios interrogantes: ¿qué había generado esta trasformación?, ¿la sola presencia de nuevos artefactos podía provocar el cambio? Además, los resultados de estos trabajos evidenciaron la existencia de tensiones relacionadas con la interpretación que los par- 
ticipantes hacen de la experiencia, el papel que otorgan a la tecnología, o las relaciones establecidas en la comunidad cuando utilizan nuevos instrumentos mediadores. Esta realidad provocó nuevas preguntas: ¿qué papel tienen estas tensiones en la trasformación del escenario?, ¿contribuyen los conflictos a crear escenarios innovadores que ofrecen respuesta a todos los estudiantes?

Intentar responder a estas cuestiones nos acercó a la teoría de la actividad (AT) cuyo origen se sitúa en la teoría histórico-cultural y la estructura jerárquica de la actividad humana de Leontiev (1978). Esta teoría interpreta la actividad humana como un sistema definido por aquellos "espacios" donde las personas adquirimos patrones propios de la comunidad a través de actividades colectivas con una estructura mediacional compleja. De acuerdo con Leontiev, la actividad humana está motivada por la necesidad de trasformar un "objeto" que puede ser material o ideal (un problema o una idea). Este motivo guía las acciones llevadas a cabo por sujetos (individuales o grupos) hacia una meta específica, utilizando herramientas propias de su cultura.

Este modelo tríadico (sujeto-herramienta-objeto) es ampliado por Engestrom (2001) en lo que él mismo denominó tercera generación de la AT, caracterizado por la importancia concedida a la actividad conjunta frente a la actividad individual y a la integración e interacción dialéctica entre todos los elementos que conforman el sistema.

$\begin{array}{ll}\text { Instrumentos: caracteristicos de la ensefianza } & \text { SISTEMA } \\ \text { formal. Discurso oral y escrito. } & \text { TRADICIONAL }\end{array}$
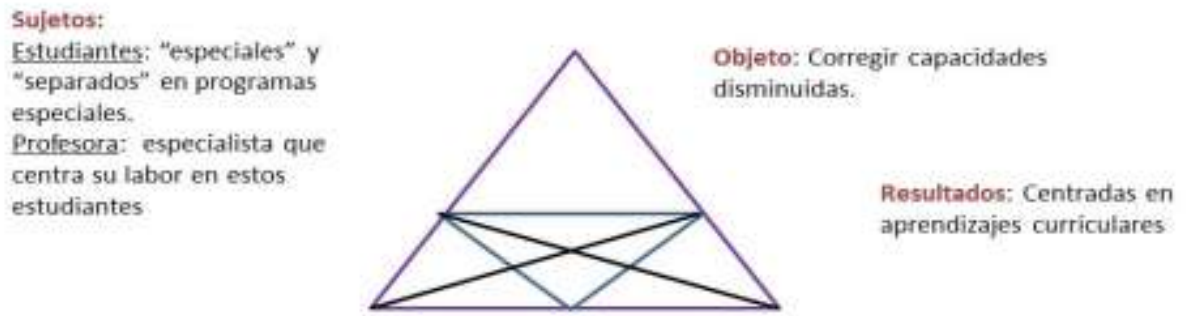

Reglas: Normas propias de la educación formal. Ideas $y$ creencias sobre las dificultades de aprendizaje, la EE y las TIC.
Comunidad: reformas y normas de División labor: expertos novatos la administración. Situación en el Conocimiento seleccionado y pautados centros de los etdiantes con nee por el adulto. Profesores especialistas que controlan lo que aprenden y cómo se aprende. 
Esta estructura nos permite explicar la actividad que se lleva a cabo en las aulas y más concretamente en un aula de apoyo, objeto de este estudio, entendida como sistema de actividad. Cada uno de los elementos representados en el diagrama (figura 1) ocupa un lugar en la estructura. El término sujeto se refiere a los estudiantes con necesidades especiales y a los profesores encargados, dentro de la comunidad, de su enseñanza. La comunidad esta formada por todo el claustro de profesores de un centro concreto o por toda la comunidad educativa, lo que tiene que ver con normas ministeriales que conforman la estructura educativa de un país, en este caso las directrices que organizan el apoyo a este tipo de estudiantes. El término división del trabajo se refiere tanto a las relaciones horizontales entre los miembros como a las verticales centradas en las relaciones de poder. En nuestro caso, definidas por las características de los estudiantes y por el papel pasivo que tradicionalmente se les otorga. Las reglas se relacionan con las normas y convenciones explícitas o implícitas que regulan las acciones y las interacciones dentro de la comunidad y que tienen que ver con las ideas acerca del aprendizaje y la enseñanza de estos alumnos y de los diferentes medios y metodologías utilizadas, incluyendo el uso de las TIC. Finalmente, los instrumentos o herramientas que se utilizan en las aulas de apoyo, se definen por su carácter histórico y cultural. En este sentido la propia historia y tradición de la educación especial hace que el instrumento más utilizado sean los textos orales y escritos, estando ausentes, casi por completo, otros instrumentos o lenguajes.

Este modelo nos ofrece una herramienta conceptual (Daniels, 2004) que, inspirada en el diálogo (formación de ideas desde diferentes perspectivas) y la multivocalidad (un reconocimiento de que las ideas y acciones son conformadas por varias voces), permite interpretar el papel de cada elemento y la interacción entre ellos. Algunos autores, incluido el propio Engestrom (2008), han utilizado también esta propuesta para analizar el contexto escolar (encontramos una buena revisión en Yamagata-Luynch, 2010). En el conjunto de estos trabajos se desprende la necesidad de construir nuevas herramientas conceptuales que tengan en cuenta el carácter dinámico de los procesos de aprendizaje y la interacción entre los diferentes elementos que, según la Teoría de la Actividad, definen el sistema donde se llevan a cabo (figura 1). En esta línea, Engestrom (2010) propone el concepto expansive learning, (EL) que sitúa en primer plano la evolución histórica y el papel del conflicto y las contradicciones en la trasformación del sistema. 


\section{La metáfora de la expansión}

El concepto de expansive learning, surge de la necesidad de explicar el movimiento inherente al proceso de aprendizaje (de la incompetencia a la competencia), y el carácter del cambio que permite dicho movimiento. Partiendo de esta idea, Engestrom (2010) platea dos cuestiones relacionadas con la naturaleza del aprendizaje: ¿se trata de un proceso que trasmite y preserva conocimiento o que lo trasforma creando algo nuevo?; ¿se trata de un proceso vertical que mejora, a lo largo del tiempo, dentro de una escala uniforme de competencias o es un modelo horizontal e hibrido que se mueve entre diferentes contextos y estándares de competencia?

La respuesta a estas cuestiones la encontramos en la metáfora de la expansión que pone el foco en la comunidad de aprendices, en la trasformación y creación de conocimiento y en los movimientos horizontales e híbridos. Desde esta perspectiva, los aprendices aprenden algo que aún no existe. Es decir, construyen un nuevo objeto en su actividad colectiva e implementan este nuevo objeto en la práctica. Para entender todas las implicaciones de esta idea, hay que tener en cuenta la distinción que esta teoría plantea entre el objeto general que surge en el sistema de actividad histórico conectado a un significado social y el objeto específico que tiene el sujeto particular en una acción determinada conectado con un sentido personal e individual.

Algunos trabajos recientes (Fujioka, 2014, Patichen y Smithenry, 2014) han tenido en cuenta estos principios a la hora de analizar los contextos educativos y su trasformación. En este sentido, la actividad que trascurre en las aulas, también las dedicadas a atender a los estudiantes con necesidades educativas especiales, se puede interpretar como una actividad colectiva que tiene sentido a partir de su evolución histórica y su vínculo con otros sistemas. Como en el resto de la actividad humana, cuando analizamos la que se desarrolla en este ámbito formal, distinguimos un nivel micro donde el actor o sujeto, en este caso estudiantes y profesores, operan con instrumentos para alcanzar objetivos a corto plazo. Estos actores a su vez, forman parte de sistemas de actividad colectivos-nivel macro-, definidos por la historia de los sistemas educativos, las teorías curriculares, o las normativas ministeriales relacionadas con la atención a la diversidad. Estos sistemas contemplan objetivos con sentido histórico y de larga duración, que entretejen las actividades individuales llevadas a cabo por los profesores y sus alumnos. En ocasiones, esta relación entre sistemas provoca tensiones que hacen que se ex- 
pandan y trasformen en algo diferente y nuevo. Este proceso de cambio será el propósito de este trabajo.

Desde este planteamiento, cuando nos acercamos a un aula donde un nuevo instrumento (por ejemplo un videojuego) convive con prácticas e instrumentos tradicionales, la unidad de análisis se define por el contraste entre dos sistemas (uno de ocio y otro formal) que comparten parcialmente el mismo objeto, y la trasformación expansiva que provoca (Engestrom y Sannino, 2010). Como veremos en la experiencia que presentamos, los participantes- profesora y estudiantes- de forma colectiva reconstruyen el objeto-problema trasformando y extendiendo el sistema de actividad, a partir de la incorporación de un videojuego, y de las contradicciones dialécticas que esta presencia provoca en otro sistema, en este caso un aula tradicional.

\section{El papel de las contradicciones}

Por la importancia que tiene en este marco de análisis, vamos a detenernos a explicar brevemente el significado de las contradicciones o tensiones en el proceso de trasformación innovadora de la actividad. El término contradicción no se tiene que entender de forma simple sólo como un problema, obstáculo, o conflicto. Por el contrario, refleja algo más complejo que indica desajustes que pueden ocurrir dentro de un solo elemento o entre varios de ellos, entre diferentes actividades o entre diferentes fases del desarrollo de la actividad (Blin y Munro, 2008). Desde este planteamiento, si queremos valorar si una experiencia innovadora en las aulas ha supuesto una trasformación del sistema, no debemos focalizar nuestro análisis sólo en la trasformación de los participantes, sino también y de forma significativa, en las modificaciones que se producen en la estructura en su conjunto y en cada uno de sus elementos. No podremos considerar una verdadera expansión del sistema si la disrupción, en el caso que analizamos en este artículo, provocada por la inclusión de un videojuego, no afecta a toda la estructura y a todos sus elementos, es decir si no impregna a todo el proceso de enseñanzaaprendizaje.

Tomando de nuevo como referencia a Engestrom (2010), las contradicciones no son algo estático que aparezcan en un momento fijo, pueden aparecer en diferentes momentos del proceso de expansión: a) como contradicciones primarias de forma latente relacionadas con 
uno o varios elementos del sistema; b) como contradicciones secundarias que aparecen entre dos o más elementos (por ejemplo entre viejos y nuevos instrumentos, como en el estudio de caso que presentamos ); c) como contradicciones terciarias existentes entre un modelo nuevo de actividad y las reminiscencias del anterior; d) y finalmente, como contradicciones que aparecen ante una nueva forma de organizar la actividad y sistemas vecinos con otras formas de organización.

Teniendo en cuenta todo lo señalado hasta el momento, la metáfora de la expansión y el papel y significado de las contradicciones en el proceso, resultan muy sugerentes cuando se quiere comprender lo que ocurre en un aula al introducir un videojuego comercial como mediador en el aprendizaje. La presencia de este medio supone establecer una relación dialéctica (figura 2) entre un sistema propio del ocio y un sistema característico de la enseñanza formal, lo que desencadena la creación de un sistema nuevo e innovador.

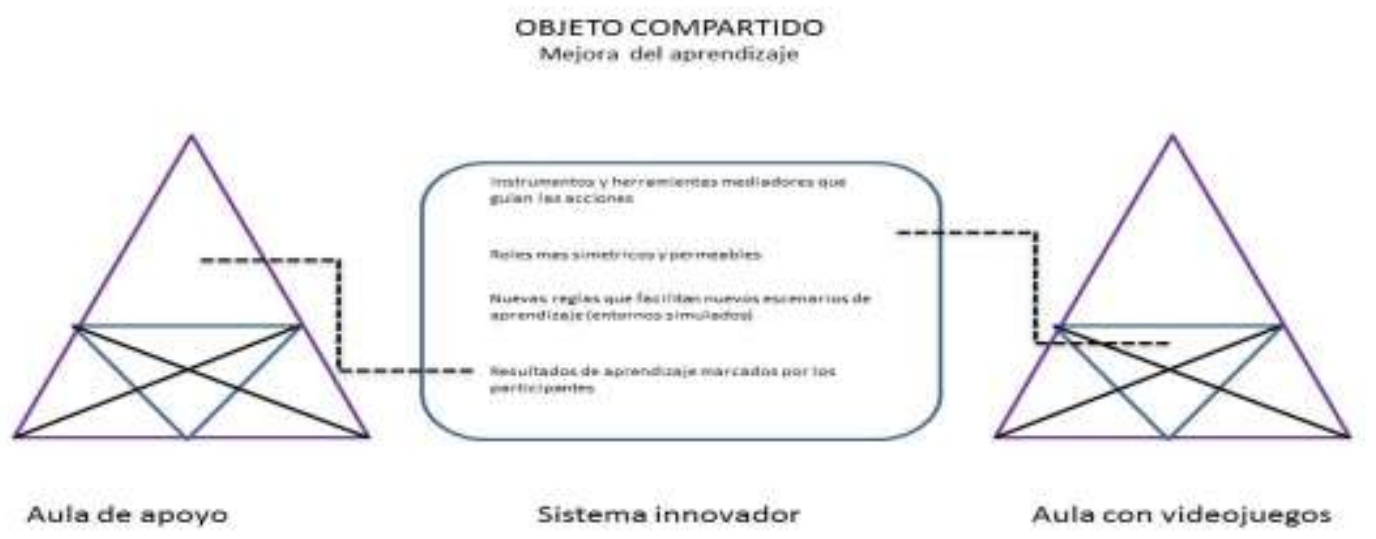

Figura 2. Tensiones entre sistemas que comparten la misma meta (adaptado de Engestrom y Sannino, 2010)

\section{Objetivos}

El objetivo de este estudio es analizar el proceso de enseñanza-aprendizaje y su trasformación cuando se incorpora un videojuego comercial (Sim3) a un aula de apoyo. Para interpretar esta transformación utilizamos como eje para el análisis los elementos que definen este espacio de aprendizaje como sistema de actividad, mostrando las tensiones y la dirección del cambio en cada uno de ellos. Este propósito general se concreta en tres objetivos específicos: 
1) Analizar el papel de videojuego como instrumento mediador en la actividad conjunta que desarrollan los participantes (profesora y estudiantes)

2) Explorar las tensiones generadas en el aula, entendida como sistema de actividad, cuando se introduce un instrumento tecnológico propio del ocio.

3)Analizar el efecto de los conflictos y tensiones entre el aula tradicional y el nuevo sistema generado con la introducción del videojuego.

\section{Método}

\section{Participantes y contexto}

El estudio se llevó a cabo en un centro público de Educación Secundaria Obligatoria (ESO) situado al sur de la Comunidad de Madrid (España). A lo largo de tres años (20092012) se implementó el uso de videojuegos comerciales como herramienta educativa, en varios cursos y con diferentes profesores.

El caso que presentamos se centra en la profesora de apoyo especialista en pedagogía terapéutica y dos grupos de estudiantes con necesidades educativas especiales (NEE). La profesora (RP) especialista en pedagogía terapéutica, posee una amplia experiencia profesional de más de 25 años. Licenciada en pedagogía, ha sido profesora de varias etapas educativas, además de asesora en centros de formación del profesorado. Ha coordinado varios proyectos de innovación educativa. Cuando realizó la experiencia llevaba 4 años en el centro.

En este estudio participaron 14 estudiantes, 6 chicos y 8 chicas entre 12 y 16 años, pertenecientes al Programa de Cualificación Profesional (PCPI) "especial”. A excepción de un estudiante con síndrome de asperger, escolarizado con anterioridad en un centro específico, todos habían estado desde el principio de su escolarización en el programa de integración. La clase de primero estaba formada por tres estudiantes diagnosticados de retraso mental, uno con retraso en su desarrollo y desfase curricular por ambiente desfavorecido y de riesgo, otro afectado de espina bífida y el tercero diagnosticado con síndrome de asperger. Por su parte, todos los estudiantes de la clase de segundo estaban diagnosticados con retraso mental, estando en uno de ellos asociado a un ambiente desfavorecido y de riesgo. Ambos grupos presen- 
taban dificultades en el aprendizaje y en el logro de tareas que implican procesos cognitivos complejos. Además, todos en mayor o menor medida adolecían de habilidades sociales y emocionales.

\section{Instrumentos}

De acuerdo con los objetivos de este trabajo, las fuentes de datos escogidas permiten situar el foco del análisis en la exploración de procesos y en la interpretación y significados que les otorgan los participantes. En concreto, utilizamos para el análisis datos con formato audiovisual con evidentes ventajas para un estudio de corte etnográfico: Entrevistas semiestructuradas grabadas con la profesora (dos iniciales, dos de seguimiento y otra al finalizar cada taller), y con los estudiantes, en pequeño grupo, al inicio y al final del taller; grabaciones audiovisuales de cuatro sesiones (dos en cada taller), fotos y sumarios de cada sesión realizados por la profesora, documentos docentes (programación y memoria de la actividad), y textos escritos de los estudiantes en el blog creado para esta experiencia.

\section{Procedimiento}

La profesora diseñó, dentro de la programación del ámbito socio-lingüístico, un taller de videojuegos. El videojuego elegido, los Sim 3, permite al jugador diseñar un personaje (definido por él mismo) y crear un escenario virtual (ciudad, barrio, familia, etc.) similar al mundo real o totalmente ficticio. El jugador trata de responder lo mejor posible a las metas derivadas de las aspiraciones de su avatar (trabajo, relaciones sociales, estado de salud, etc), para ello deberá tomar decisiones y actuar dentro de unos parámetros y reglas.

El taller se llevó a cabo con dos grupos de estudiantes de $1^{\circ}$ y $2^{\circ}$ del PCPI "especial" dentro de la programación del módulo formativo de carácter general y más concretamente dentro del ámbito socio-lingüístico. Cada taller se desarrolló en 6 sesiones llevadas a cabo durante cuatro meses de un mismo curso académico. En la tabla se reflejan los objetivos y actividades de cada sesión comunes a los dos talleres. 
Tabla 1. Objetivos y actividades en cada sesión

\begin{tabular}{|c|c|c|}
\hline Sesiones & Objetivos didácticos específicos & Actividades \\
\hline 1 & $\begin{array}{l}\text { Presentación del taller; Descubrir las ideas previas de los estudiantes sobre } \\
\text { los videojuegos; Conocer su experiencia con los videojuegos; Presentar los } \\
\text { objetivos del taller y el videojuego. }\end{array}$ & $\begin{array}{l}\text { Diálogo en gran grupo } \\
\text { Visionado de un tutorial del } \\
\text { videojuego }\end{array}$ \\
\hline 2 & Entender el concepto de descripción & $\begin{array}{l}\text { Diseñar los avatares en } \\
\text { pequeño grupo } \\
\text { Describir verbalmente el } \\
\text { aspecto físico de cada avatar } \\
\text { (rasgos físicos y formas de } \\
\text { vestir). Además, describían su } \\
\text { avatar en el blog creado para } \\
\text { esta experiencia. }\end{array}$ \\
\hline 3 & Entender el concepto personalidad y los elementos que la definen. & $\begin{array}{l}\text { Definir la personalidad de los } \\
\text { avatares y sus gustos y } \\
\text { aficiones. Describir } \\
\text { verbalmente y en el blog el } \\
\text { aspecto físico de cada avatar } \\
\text { (rasgos físicos y formas de } \\
\text { vestir). }\end{array}$ \\
\hline 4 y 5 & $\begin{array}{l}\text { Tomar conciencia del proceso del juego y de los problemas que surgen } \\
\text { durante la partida y la forma de resolverlos. Trabajar conceptos que van } \\
\text { surgiendo durante la partida como tipos de trabajo y su relación con las } \\
\text { características de los avatares; hábitos de consumo y uso del dinero en la vida } \\
\text { cotidiana, etc. Desarrollar la capacidad de expresión escrita y la elaboración } \\
\text { de narrativas. }\end{array}$ & $\begin{array}{l}\text { Desarrollo del juego y de la } \\
\text { partida } \\
\text { Después de las sesiones de } \\
\text { juego, los estudiantes escribían } \\
\text { en un blog creado para esta } \\
\text { experiencia. }\end{array}$ \\
\hline 6 & $\begin{array}{l}\text { Elaborar narrativas orales relacionadas con los procesos de juego. Tomar } \\
\text { conciencia de los procesos de aprendizaje que han tenido lugar, relacionados } \\
\text { con procesos cognitivos (resolución de problemas) y sociales (trabajo } \\
\text { colaborativo, comunidad de aprendizaje) }\end{array}$ & $\begin{array}{l}\text { Discusión grupal y elaboración } \\
\text { de narrativas orales colectivas. }\end{array}$ \\
\hline
\end{tabular}

Además de los objetivos específicos (tabla 1), la programación elaborada por la profesora contempla cuatro objetivos trasversales incluidos dentro del ámbito socio-lingüistico y que se desarrollan a lo largo de todas las sesiones: desarrollar procesos de pensamiento utilizando recursos tecnológicos; desarrollar la capacidad de aprender a aprender a través del uso de recursos y herramientas que nos ofrecen las TIC; favorecer el diálogo y la argumentación utilizando escenarios multimedia; potenciar la autoestima de cada miembro del grupo al tiempo que aprenden a cooperar para conseguir un objetivo común.

Las sesiones tuvieron una duración de 80 minutos. Cada una de ellas se organiza en torno a tres fases: En la primera $(10 \mathrm{~m}$.) se realiza una discusión grupal en la que se abordan los objetivos de la sesión, a partir de la revisión de lo realizado en la sesión anterior. En la segunda, distribuidos en pequeño grupo, se desarrolla el juego durante $60 \mathrm{~m}$; la última fase se dedica a discutir de nuevo en gran grupo y revisar lo que ha ocurrido en la sesión, los logros y 
las dificultades que han surgido, teniendo en cuenta los objetivos que los participantes se habían marcado. Después de las sesiones de juego los estudiantes, con ayuda de la profesora, escribían en el blog, creando pequeñas narraciones, con la descripción de su avatar y la vida que le había diseñado.

Los talleres se realizaron en el aula de apoyo, equipada con dos consolas wii, una pantalla de televisión y un monitor de PC. La profesora distribuyó a los estudiantes en dos pequeños grupos atendiendo a sus diferentes capacidades, con el fin de que pudieran compensarse y trabajar de forma colaborativa. El número de estudiantes en cada grupo osciló entre dos y cuatro.

\section{Diseño y análisis de los datos}

El estudio de caso que presentamos ha sido analizado desde una metodología cualitativa siguiendo un enfoque etnográfico de estudio de casos (Hamera, 2011; Yin, 2011). Un aspecto a tener en cuenta es el hecho de que estos trabajos no basan su validez en la frecuencia de aparición de cierto fenómeno, sino en la descripción detallada de casos en los que se pueda explicar cómo las personas atribuyen sentido a su actividad en contextos socioculturales definidos. Esta forma de investigar implica la utilización de diversas fuentes de datos que ayuden a interpretar las rutinas y situaciones problemáticas en la vida de las personas desde los significados que ellos mismos les otorgan. El caso objeto de este artículo forma parte de un proyecto más amplio realizado por el equipo de investigación, Imágenes, Palabras e Ideas.

Este estudio lo abordamos desde una doble vertiente: a) La perspectiva del cambio. No nos interesa ofrecer una foto fija de lo que ocurre en el aula. Por el contrario, estamos interesados en las tensiones y conflictos que esta presencia ha generado en el contexto tradicional y cómo su superación ha llevado a la creación de un escenario innovador de aprendizaje; b) la perspectiva de los participantes. Para llevar a cabo este objetivo, nuestra principal fuente de datos son las interpretaciones de los propios participantes, sujetos y comunidad en la terminología de la AT. 


\section{Resultados}

Como hemos venido señalando, el modelo diagramático que define la AT, nos ha permitido comprender, organizar e integrar los datos cualitativos obtenidos en este estudio de caso. En la experiencia que analizamos el aula ordinaria de apoyo sufrió una serie de cambios cuando la profesora decidió introducir el videojuego de los SIM. La aparición de este instrumento tecnológico provocó la expansión del sistema y la creación de algo diferente.

De acuerdo con los objetivos de este trabajo, los diferentes elementos del sistema nos servirán para organizar la exposición de los resultados. Con relación a cada uno de ellos exploramos, a partir de las interpretaciones de la profesora y los estudiantes, las tensiones y contradicciones surgidas entre un sistema tradicional y un sistema donde se ha introducido un artefacto nuevo, como el videojuego.

\section{La razón del cambio. El objeto de la actividad}

Una de las primeras tensiones que surgieron cuando la profesora propuso llevar a cabo un taller de videojuegos, fueron las diferentes interpretaciones acerca del propósito de la actividad, dentro de la comunidad educativa, y entre los participantes, estudiantes y profesora (figura 3).

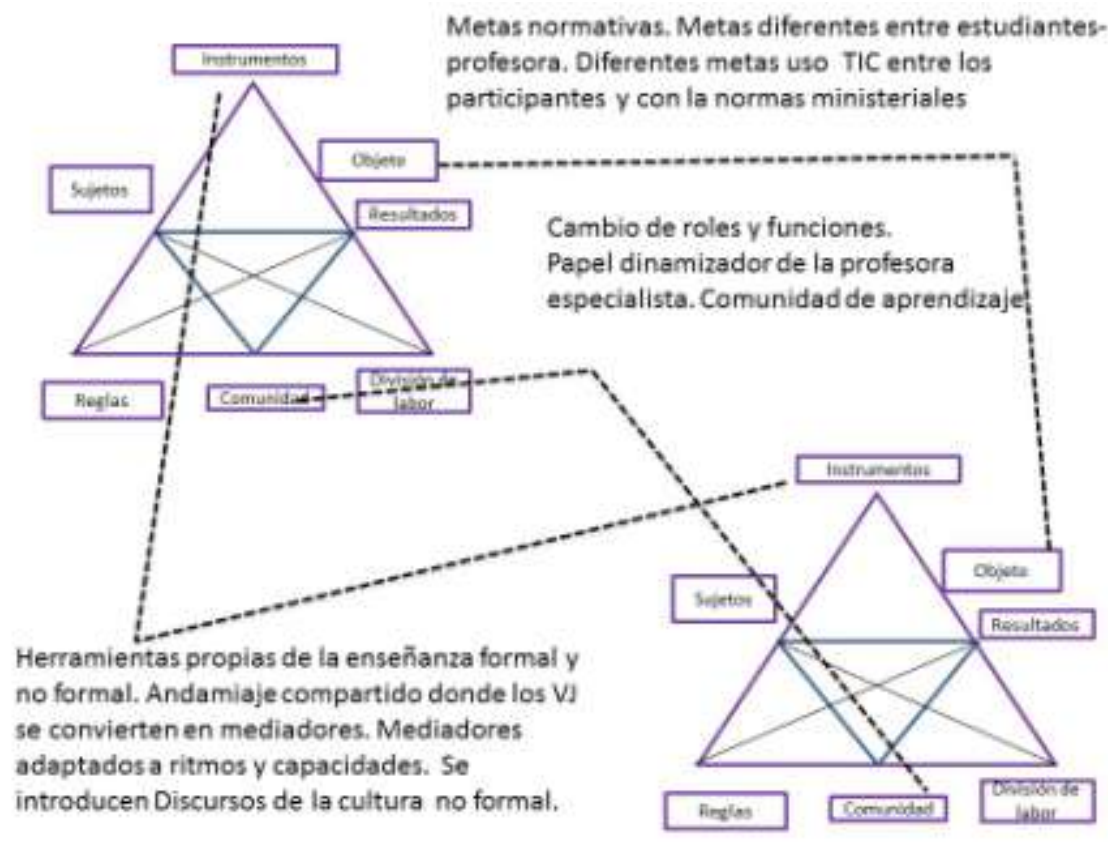

Figura 3. Tensiones y contradicciones del sistema (I) 
Algunas de estas tensiones se relacionan con el objeto de la actividad colectiva y con la interacción con otros sistemas externos, otras tienen que ver con la meta de la actividad y las acciones individuales de los participantes y sus motivaciones a corto plazo (Daniels, 2004). La presentación del taller a la dirección del centro y al claustro de profesores creo tensión entre diferentes perspectivas, la de la profesora, la de algunos colegas y la corriente social que demoniza a este medio.

(Primera entrevista de seguimiento). Cuando comenzaron los talleres de videojuegos hubo profesores que no quisieron, porque no lo veían, no veían las posibilidades que podía tener..., preparadas.., muchos decían "es una pérdida de tiempo"..., y el "cómo vamos a meter una cosa a la que están enganchados, para que se enganchen más”... Además cuando empezamos, en aquella época, había un rechazo social a los videojuegos, la idea era que los chavales están durante horas con ellos, y que no se relacionaban con otros niños.

En contraposición a la opinión de sus compañeros, para RP el videojuego sí posee posibilidades educativas claras. Su propia experiencia como jugadora y la colaboración con el equipo de investigación en experiencias anteriores la habían convencido de esto. Para superar esta tensión se realizaron reuniones formales e informales a lo largo del proyecto con los profesores, y se organizaron visitas de algunos de los talleres de videojuegos. Estas acciones contribuyeron, como nos explica RP en la primera entrevista de seguimiento, a superar la tensión y a que algunos compañeros cambiaran su creencia sobre los videojuegos y sus posibilidades educativas. Este cambio de actitud supuso una expansión del sistema hacia propuestas más innovadoras consensuadas y compartidas por la comunidad educativa.

(Primera entrevista de seguimiento) La idea que cambio, es que se viera que trabajar con los videojuegos era algo más que jugar, de que podía colaborar en una tarea común, el hecho de que no eran juegos individuales, sino grupales, que había que tomar decisiones compartidas ..., Eso algunos compañeros lo veían... y eso les convencía.

Estas no fueron las únicas tensiones. También (figura 3) surgieron contradicciones con otros sistemas externos al centro y relacionados con la administración educativa. Una buena muestra fue la visita a uno de los talleres del asesor de zona para la formación de profesores.

(Segunda entrevista de seguimiento) El asesor vino a visitar uno de los talleres y presentó un informe negativo diciendo que no había ninguna actividad formativa, que solo juga- 
ban..., él no veía que hubiese ningún trabajo de programación ni formación del docente para implementar esa actividad,..., para él solo era un juego...

La contradicción entre la forma de interpretar la actividad del asesor y de la profesora es un buen ejemplo de lo que Engestrom (2009) denomina "multivocalidad" donde conviven diferentes "voces", cuya presencia provoca una contradicción entre lo que hay que hacer (discurso político-administrativo) y lo que se hace (discurso de la práctica) (Larripa y Erausquin, 2010). Ante esta contradicción, la profesora hace prevalecer el discurso de la innovación educativa, reinterpretando la norma y apoyándose en una definición comprensiva de la "competencia digital", término contemplado en los documentos ministeriales. Desde esta perspectiva, como relata en la última entrevista, considera el videojuego un buen recurso para desarrollar habilidades cognitivas y sociales.

(Entrevista final) Para mí estaba claro, esta era una manera muy cerrada de interpretar una competencia recogida en el currículum y en la normativa, donde se habla de competencia digital... y yo me acojo a ello..., competencia digital no es solo saber utilizar el Word o buscar información..., las Tic sirven para más cosas, también los videojuegos con los que pueden aprender muchas cosas, enfrentarse a problemas, relacionarse o elaborar historias con las que expresarse.

Por su parte, a lo largo de la experiencia, y coincidiendo con otros trabajos (Swain, 2011) pudimos observar cómo se generaban también tensiones entre las diferentes metas y motivaciones que tenían los participantes en los talleres. A la motivación de la profesora antes mencionada, hay que confrontar la de los estudiantes. La mayoría de ellos consideraban el taller de videojuegos como una oportunidad para jugar y divertirse, lo que llevó a algunos estudiantes a rechazar la idea de utilizarlos en el aula.

(Entrevista final). Aunque tú se lo expliques..., yo siempre se lo explicaba, que no solamente era jugar, que íbamos a trabajar otras cosas, pero yo creo que ellos lo que ven es el juego nada más... Mira, hubo dos reacciones muy diferentes, una la de los que ya habían jugado que dijeron “ique bien;”... y otros que no les gustaba la idea. Por ejemplo J cuando lo plantee, me dijo: J: "Profe, para qué, ya tenemos muchas cosas que hacer..., no vamos a ponernos a jugar, aquí venimos a trabajar...". 
Que los estudiantes lo vean solo como un juego entra en contradicción con el propósito de la profesora centrado en que sus estudiantes tomen conciencia y sean protagonistas de su propio aprendizaje. Este fue uno de los principales desafíos para el nuevo sistema que se superó, en parte, por dos actividades introducidas por la profesora: dialogar y reflexionar juntos, al finalizar cada sesión, sobre lo que habían hecho durante el juego; y participar en el blog describiendo su avatar y narrando su vida virtual. Ambas actividades les ayudó a reelaborar su idea sobre la actividad, yendo más allá del juego, y tomando conciencia de lo aprendido durante el taller.

(Entrevista Grupo $2^{\circ}$ ) Investigador: ¿Se aprende jugando con tu avatar y los videojuegos?, -A1: Pues se aprende a vivir compartiendo la casa, porque estamos nosotros tres compartiendo la casa. Y uno está durmiendo y el otro está mirando para dormirse al lado..., necesitamos aprender a vivir juntos- Investigador: ¿y tú, qué has aprendido?- A2: Se aprende a... es una visión de lo que es la vida real. En cierta manera es la vida real, pero... es una... es como la vida real, pero con más diversión.

Estas respuestas muestran cómo las metas de la profesora y estudiantes se acercaron llegando a compartir un objeto común, en este caso, un espacio para el aprendizaje.

\section{Los participantes y su papel en la trasformación}

Otro de los elementos que generó tensión está relacionado con la identidad y lugar que ocupan en la comunidad educativa los sujetos, en este caso estudiantes con necesidades educativas especiales. Como ya vimos antes, el discurso "correcto" de la inclusión educativa (lo que tiene que ser), entra en colisión con algunas prácticas herederas de modelos tradicionales. Esta contradicción es lo que manifiesta la profesora cuando se le pregunta sobre cómo ve la comunidad educativa a sus estudiantes.

(Primera entrevista inicial). Para el centro estos estudiantes forman un grupo aparte, son el grupo "especial", tienen como ese título... En general a estos alumnos se les trata, casi siempre, desde lo que no pueden hacer..., y ellos también se sienten así, porque a lo largo de su escolaridad han vivido frecuentes situaciones de fracaso que ha hecho que se sientan poco capaces y que los demás les vean con pocas posibilidades.

Como describe RP, a pesar de las normas favorables a la inclusión educativa, estos estudiantes son identificados por la discapacidad que les dificulta el acceso a los contenidos curriculares. Esta creencia les coloca en un lugar definido por su trastorno y por su inclusión en 
programas específicos de enseñanza. El diseño del taller y la introducción del videojuego en el aula, supuso una expansión del sistema hacía otros referentes competenciales. En el nuevo sistema, heredero de un sistema de ocio, la competencia se mide por la superación de los retos y problemas planteados durante las partidas.

(Sumario, $2^{a}$ sesión, grupo $1^{o}$ ) Hoy no ha venido A, y L ha montado sola la consola, se muestra muy satisfecha de haberlo logrado, sin ayuda

En este extracto del sumario de la $2^{\mathrm{a}}$ sesión observamos que una estudiante ("L") ha desarrollado una nueva competencia que le permite realizar de forma autónoma una tarea (montar la consola) que antes realizaba con la ayuda de otra compañera ("A") más experta. Mientras que en el sistema tradicional las tareas requieren un grado competencial fijo, en el taller las competencias son más abiertas y flexibles. En este sistema, el grado competencial se mide con una doble referencia, la representación que tiene el jugador de su grado competencial y la que le otorgan los otros jugadores.

(Grabación $3^{a}$ sesión. Grupo $1^{o}$ ) Profesora: ¿Compró ella la nevera?- A 3: Sí - A2: Yo no la compré, fueron ellos... - A3: No, profe. Yo se lo expliqué y la compró ella.- A2: Ah, ah... es verdad, al final la compré yo... -Profesora (dirigiéndose a A2): pues intenta comprar ahora la cama - A2: vale, ahora voy

En esta trascripción de la tercera sesión, un estudiante (A2) duda de su propia capacidad para comprar muebles. Esta idea entra en contradicción con la visión de sus compañeros que piensan que sí es capaz de hacerlo (A3). La representación que sus compañeros de juego tienen sobre su competencia, unido a las experiencias de éxito que ha tenido a lo largo de las sesiones, le ha dado la oportunidad enfrentarse y superar nuevos retos (alejados del currículum tradicional), aumentando la confianza en sí misma.

Esta trasformación de las referencias competenciales provocó también un cambio en la distribución de roles, otorgando a los miembros de la comunidad diferentes funciones y responsabilidad en la actividad. En el aula de apoyo, antes de la incorporación del videojuego, la estructura relacional, heredera de un modelo tradicional, situaba al profesor en el centro asumiendo el papel único y exclusivo de experto, frente a los estudiantes.

(Segunda entrevista de seguimiento) En las clases es el profesor el que decide, ellos no saben manejar iniciativas por sí mismos, tienen una visión muy distorsionada de sus habi- 
lidades y los profesores tampoco creemos que puedan participar en su propio aprendizaje..., ante iniciativas o actividades nuevas siempre dicen: no puedo, no sé.

Como expresa RP en la entrevista inicial, en un aula tradicional el profesor organiza el aprendizaje, imponiendo el guion de las actividades, lo que incluye la secuencia de acciones o la selección de instrumento (Heemskerk, Volman, Dam, y Admiraal, 2011). En este sistema los estudiantes asumen un rol pasivo participando de forma muy periférica en el proceso. Este modelo entra en contradicción (figura 4) con la nueva estructura de los talleres donde la inclusión de una práctica de ocio, como jugar con videojuegos, supone una trasformación de las relaciones de poder establecidas en el aula y del significado que cada sistema, el tradicional y el innovador, otorga a expertos y novatos.

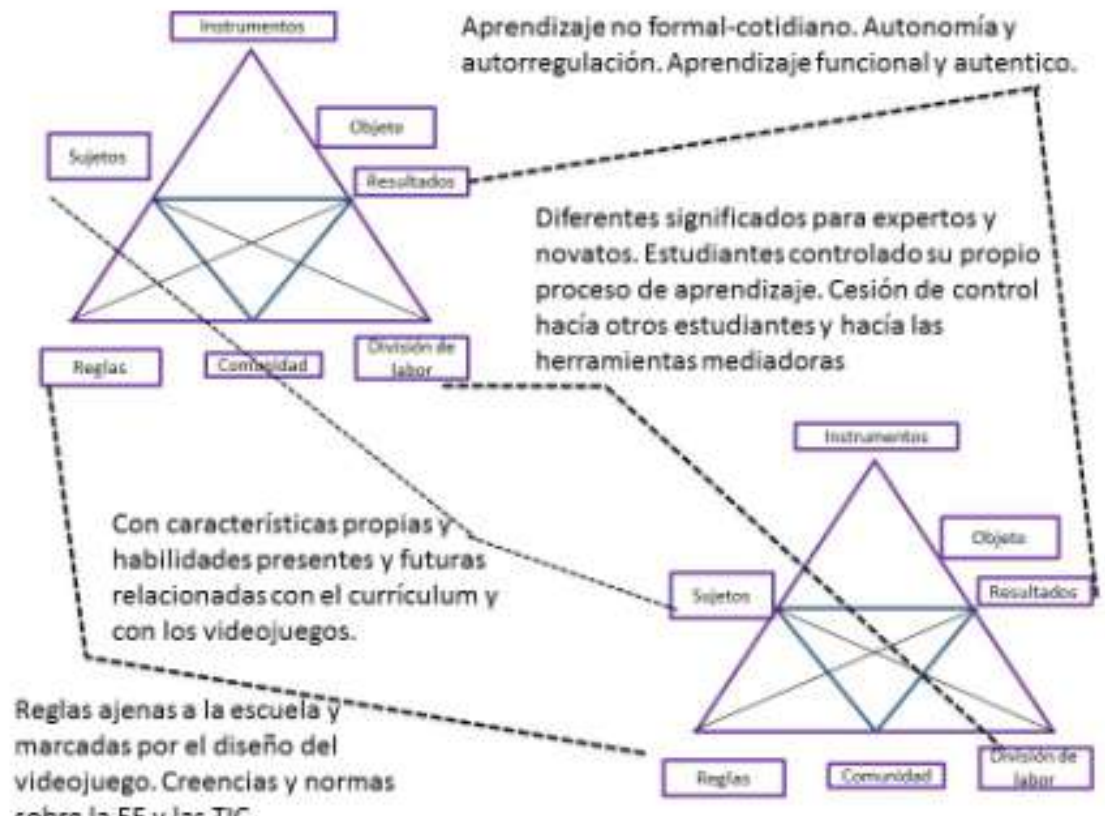

sobre la EE y las TIC.

Figura 4. Tensiones y contradicciones del sistema (II)

La dirección del cambio. Los artefactos y reglas del sistema

Dos son los elementos que guían y conducen el proceso de expansión del sistema, transformándolo en algo distinto: los artefactos que median entre los sujetos, el objeto y los resultados de la actividad y las reglas y normas que delimitan estas actividades.

En la entrevista RP explica el tipo de instrumentos utilizados en las aulas de apoyo, donde el discurso oral y escrito es el principal mediador en el aprendizaje, excluyendo materiales e instrumentos que utilizan otros lenguajes y códigos. 
(Segunda entrevista inicial) Normalmente, se plantea como una clase tradicional, hay un material para ellos, fichas, libros concretos con adaptaciones curriculares, contenidos delimitados, actividades con la estructura de siempre... Lo que más se hace son actividades sobre papel..., la meta es cumplir los objetivos, aprender unos contenidos.

La experiencia adquirida a lo largo de los años, han convencido a RP de la necesidad de introducir nuevos instrumentos y de diseñar un nuevo sistema donde convivan herramientas tradicionales y nuevas.

(Sumario, $2^{a}$ sesión, grupo $2^{\circ}$ ) Iv comenta en alto la dificultad del juego "yo no me entero de nada profe". Le propongo comprobar para qué sirven los botones de los mandos y los iconos que aparecen en la pantalla. Analizamos uno a uno para que entienda su significado, y comienza a sentirse más seguro, "esto va profe”...

En este extracto del sumario de la $2^{\mathrm{a}}$ sesión podemos observar que la mediación no se centra exclusivamente en el profesor y su discurso oral, sino que se distribuye entre otras "voces" y herramientas. La profesora deja que los elementos del propio instrumento sirvan de ayuda para que Iv consiga sus metas.

(Grabación, $4^{a}$ sesión, grupo $1^{o}$ ) Uno de los alumnos, cuando le toca en turno, intenta meter a su avatar en el dormitorio. sin éxito. A: no sé cómo se hace... (le da el mando a la profesora). $P$ : (la profesora le devuelve el mando) presiona aquí y dirígelo hacia la pantalla, hacia la derecha... (la profesora se pone a su lado). Qué crees que le pasa, por qué no avan$z a$ ? (señala a la pantalla, donde sale un triángulo, símbolo que indica el estado del avatar) mi$r a$, a ver qué te está diciendo... A: es que tiene sueño, necesita dormir, entonces tengo que buscar una cama...
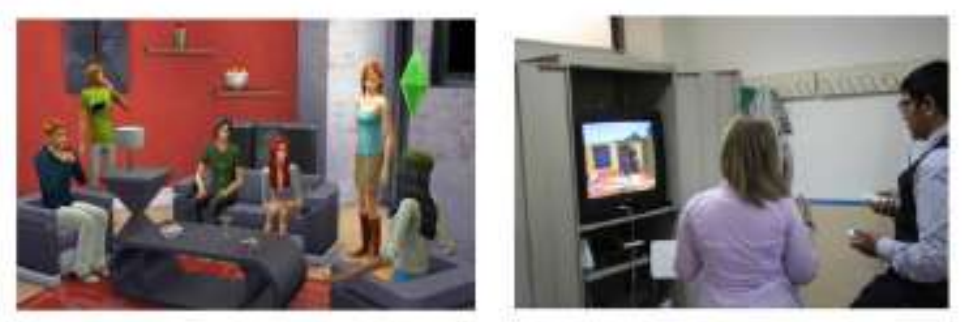

Figura 5. Herramientas y signos que median en la actividad

En esta trascripción del diálogo observamos de qué forma, con la ayuda de la profesora, los iconos que aparecen en la pantalla (un rombo que indica el estado del avatar, figura 5) 
o los signos dibujados en el mando ayudan a este estudiante indicándole lo que puede o no puede hacer, y mostrándole cada una de las posibilidades de acción. De esta forma, la profesora no es la única ayuda que reciben los estudiantes, los nuevos instrumentos, el videojuego y los signos y símbolos que conforman su lenguaje, se han convertido en un elemento más para "andamiar" el aprendizaje.

La interpretación de la forma en que el videojuego ha mediado en la actividad no sería completa sin hacer referencia a las contradicciones surgidas alrededor de las reglas y normas que regulan el uso de las TIC, en general y del videojuego en particular. Dentro de una perspectiva correctora, que define los sistemas tradicionales de apoyo, encontramos dos enfoques que determinan el uso de la tecnología (Abbott, 2007, 2011; Monjelat y Méndez, 2012). En uno se las considera un recurso para entrenar habilidades, mientras que en otro la tecnología sirve para permitir el aprendizaje. En esta entrevista, la profesora reconoce la convivencia en el aula de los dos enfoques señalados más arriba como formas de interpretar las relaciones entre los recursos tecnológicos y el aprendizaje.

(Segunda entrevista inicial) Sí, alguna vez los he usado... son programas para entrenar habilidades de cálculo, son un complemento a otras actividades, quizá más motivadores..., como trasladar los ejercicios del libro a un entorno más atractivo. La ventaja quizá es que el error se ve de otra forma, no tan cerrada ni evidente. Bueno también en algún caso, utilizamos las TIC para.., el ordenador con algunos programas informáticos les permiten el acceso a las tareas, como cuando tienen dificultades visuales y les podemos ampliar la letra.

Por otra parte, la presencia de los SIM introduce las reglas propias del juego (figura, 5) determinadas por su diseño y por el mundo virtual creado. Así como los textos tradicionalmente utilizados en la escuela tienen sus propias reglas, el videojuego tiene otras definidas por los entornos virtuales que permiten o limitan las acciones de los jugadores (Gee, 2007). En este universo los estudiantes/jugadores pueden "simular" una vida en un entorno social ficticio. Para conseguirlo, el videojuego ofrece nuevas herramientas y códigos (imágenes, sonidos, o simulaciones), para que interpreten el mundo y actúen sobre él.

(Entrada en el blog, grupo $1^{\circ}$ ). Me llamo Ken tengo 27 años y ahora estoy con unos amigos viviendo. Soy una persona callada y valiente, me gusta hacer deporte libre, natación y comer mucho. 
En el mundo simulado de los Sim, el autor de este texto, puede representar un "yo" ficticio. A través del avatar creado proyecta su deseo de llevar una vida independiente, viviendo con amigos. Una vida a la que, por su discapacidad, no puede tener acceso.

(Entrevista Grupo $1^{o}$ ) Investigador: ¿El avatar es como si fueras tú? - Al: Claro.- Investigador: Cuéntanoslo otra vez. - Alumno: Pues me llamo Ken, aunque no me llamen Ken, me llamo xxx; tengo 27 años, aunque tengo 18; vivo con un amigo... - Investigador: Con un amigo? - Al: Me gusta hablar con él, charlar, me gusta comer con los amigos, alitas de pollo al ajillo, todo eso me gusta.

En esta entrevista final, este mismo estudiante, con una discapacidad acusada en el ámbito de la relación social, explica, en primera persona, cómo vive y se relaciona a través de su avatar

\section{Las consecuencias del cambio. Los resultados del sistema}

Finalmente, nos fijamos en los resultados de la actividad, elemento del sistema donde se generan contradicciones y tensiones importantes. En el caso que estamos analizando, un curso de PCPI “especial” (ámbito socio-lingüístico), la administración educativa, a través del currículum, marca las competencias que tienen que desarrollar los estudiantes, relacionadas con el uso del lenguaje como medio de expresión y de relación con el mundo. Aunque tal y como refleja la normativa, la competencia hace referencia a diferentes formas de expresión, en la cultura docente dominante, como relata RP en el extracto de entrevista, impera un interés por los componentes gramaticales del lenguaje.

(Entrevista final) El caballo de batalla de todos los profesores es la parte gramatical, saber los pronombres personales.., si estás en primera persona..... Justamente se pone el acento en lo que les cuesta más y además les sirve menos... siempre se está enseñando lo mismo, y eso se ha visto que no funciona,...

Este interés por los conocimientos gramaticales entra en contradicción con el propósito de la profesora, interesada en dotar a los estudiantes de competencias para expresarse y relacionarse a través de diferentes formas y códigos.

(Entrevista final) A mí me interesa la expresión oral, que se expresen ..., no me interesa tanto la parte gramatical, eso lo dejo aparte, que es lo que viene en el currículum oficial,..., a mí me interesa que ellos sean capaces de expresarse de múltiples formas que les permita enfrentarse, a situaciones personales y profesionales, por ejemplo atender a un visitante, si acaban siendo bedeles... 
Esta tensión provocó que la profesora decidiese introducir nuevos lenguajes y formas de expresión. La presencia del videojuego o del blog cambio la tendencia dominante, permitiendo que los estudiantes se expresaran combinando lenguajes (texto escrito e imagen) en la construcción de narrativas, basadas en entornos simulados, como nos muestra (figura, 6) el texto y la captura de pantalla que un estudiante subió al blog.
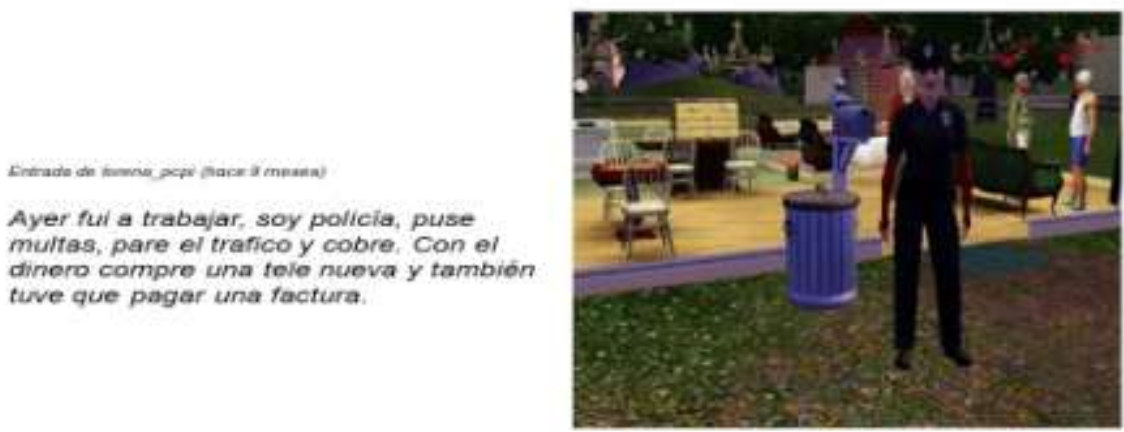

\section{Figura 6. Construyendo narrativas en el blog}

Respecto a la naturaleza de los resultados, durante la experiencia convivieron los esperados en el contexto formal, relacionados con contenidos curriculares y tareas de solución única, con los resultados esperados cuando jugamos con un videojuego. La inclusión de un objeto propio de la cultura del ocio supuso un cambio en la naturaleza de los resultados permitiendo a los jugadores adoptar diferentes soluciones ante un mismo problema. En la siguiente trascripción vemos cómo ante la necesidad de encontrar trabajo y obtener dinero para vivir, dos grupos de estudiantes utilizaron estrategias diferentes para llegar a la solución.

(Grabación $3^{a}$ sesión, grupo $2^{\circ}$ )- Profesora: y vosotros que hicisteis para buscar trabajo- A1. Entramos en las tiendas para ver si necesitaban a alguien-Profesora (dirigiéndose a otro grupo): y vosotros - A2: Nosotros lo buscamos por internet

Por otra parte, el aprendizaje que los estudiantes realizan en el entorno simulado del videojuego tiene una consecuencia inmediata y funcional, a diferencia de lo que ocurre en la enseñanza formal donde los resultados del aprendizaje son poco significativos para el apren- 
diz. El siguiente diálogo entre la profesora y dos estudiantes refleja cómo están aprendiendo a solucionar problemas reales y presentes en su vida cotidiana.

(Grabación $3^{a}$ sesión, grupo $2^{\circ}$ ) Profesora: ¿Pero cuál es el problema- A3: las camas - Profesora: Espera, a ver... ¿quién ha comprado las camas? - A2: Yo, compré sólo una.Profesora: ¿Y por qué compraste una cama sólo? - A3: Claro, podría haber comprado tres, y mientras tanto nosotros comprar la comida del avatar. Claro, no lo hizo así porque no quiso. - RP: si erais tres viviendo en la casa por qué compraste sólo una cama? - A2: Porque yo pensé en mí, no en ellos. (risas) - Profesora: A ver, el problema ha sido que has pensado sólo en ti.

Como consecuencia, los resultados o productos de la actividad cambiaron, evitando focalizar la atención de forma exclusiva en conocimientos curriculares y propios de la educación formal, para mirar hacia el conocimiento cotidiano que les prepara para la vida y les facilita una mayor autonomía, autorregulación y control sobre su propio proceso de aprendizaje.

\section{Discusión}

¿Qué ocurre cuando introducimos en la escuela un instrumento propio del ocio? En este estudio hemos intentado responder a esta pregunta centrando nuestro interés en la perspectiva del cambio y el proceso de trasformación que un videojuego, instrumento ajeno al contexto formal, puede provocar en el proceso de enseñanza-aprendizaje. Tres ideas han guiado nuestro análisis. La idea de la actividad humana como un sistema definido a través de actividades con una estructura mediacional compleja (Leontiev, 1978). La idea del aprendizaje como un movimiento hacia el cambio caracterizado por la integración e interacción dialéctica entre todos los elementos que conforman el sistema (Engestrom, 2001, 2010). La tercera idea, interpreta la actividad que trascurre en las aulas como una actividad colectiva que tiene sentido a partir de su evolución histórica y su vínculo con otros sistemas (Fujioka, 2014, Yamagata y Luynch, 2010). Esta relación entre sistemas provoca tensiones que hace que se expanda y trasforme en algo diferente y nuevo (Engestrom, 2001).

Partiendo de estas ideas-eje, el primer resultado que queremos resaltar se refiere a la meta u objeto de la actividad. Durante el análisis comprobamos que incluir un instrumento tecnológico excluido del repertorio clásico de las TIC, tanto en la cultura docente como en las nor- 
mas ministeriales, provocaba tensiones entre sistemas dentro y fuera del centro (Cross, 2010). Sus propios compañeros no comprendían la decisión tomada por RP, ni tampoco lo hacían los representares ministeriales. Ambos colectivos no compartían las posibilidades que la profesora veía en el videojuego. Este resultado señala la convivencia de modelos reflejados en diferentes "voces y discursos", (Daniels, 2004; Fujioka, 2014) como el político-administrativo o el de la práctica docente, lo que exigió, como hemos visto en los resultados, acciones de acuerdo y negociación. A lo largo de las sesiones pudimos observar que la superación de esta tensión, contribuyó a trasformar el sistema en un escenario donde el videojuego trascendió su significado, vinculado al ocio, para convertirse en un artefacto mediador y trasformador del contexto de aprendizaje.

Otro resultado interesante y especialmente significativo en el modelo de análisis planteado, (Oswald,2014; Phama y Renshawa, 2015) se relaciona con los participantes, la interacción entre ellos y su papel en la actividad. En el nuevo sistema creado con la introducción del videojuego, y coincidiendo con los resultados de otros trabajos (Saljo, 2010), aunque el profesor sigue siendo una guía fundamental, la presencia del videojuego provocó una trasformación en la interacción social del aula y en el papel que asumen los participantes. Un cambio metodológico y la incorporación de un nuevo instrumento, ofreció a los estudiantes la oportunidad de asumir el papel de expertos, provocando la aparición de procesos de andamiaje entre iguales y aprendizaje cooperativo (Engestrom, 2014). La superación de un reparto de roles estricto y fijo, trasformó el aula en un espacio innovador, con una mayor permeabilidad en los roles y una interacción más simétrica entre los estudiantes y su profesora, la cual adoptó fundamentalmente un papel dinamizador.

Otro elemento que, sin duda, tiene un papel muy relevante, tal y como indican los resultados, se refiere a los artefactos y su significado mediacional en la actividad conjunta que desarrollan los participantes. A lo largo de las sesiones observamos la expansión del sistema explicada, de acuerdo con otros trabajos (Engestrom, 2010; Watson, Mong y Harris, 2011), por la convivencia de viejos y nuevos artefactos mediadores que puede provocar contradicción entre diferentes concepciones, en este caso, sobre el uso de la tecnología en las aulas. La profesora y los propios estudiantes tuvieron que modificar sus concepciones sobre la presencia de instrumentos tecnológicos como meros facilitadores en la adquisición de habilidades para considerarlos mediadores y promotores de escenarios innovadores de aprendizaje. A esto 
hay que añadir el propio carácter del instrumento introducido, un artefacto característico de un sistema de ocio. Pudimos observar en las diferentes sesiones de juego como los SIM se convertía en un instrumento mediador y determinante en el proceso de expansión y cambio del sistema y de sus diferentes elementos. Esta mediación no se entendería sin hacer mención a las reglas y normas que impone las características de este videojuego. Los SIM introducen en el aula un nuevo código de comunicación y expresión (Gee, 2010, Méndez, Lacasa, y GarcíaPernia, 2013) conformado por lenguajes, normalmente ausentes de las aulas, como la imagen, el sonido o la simulación. Este planteamiento nos ha llevado a trascender la perspectiva del videojuego como herramienta tecnológica que asiste o facilita el aprendizaje, para considerarlo un objeto cultural a través del cual se incorporan al aula nuevas prácticas, códigos y lenguajes que median la actividad y transforman todos los elementos del sistema.

Finalmente, las características del videojuego se han reflejado también determinantes en la transformación de los resultados o consecuencias del cambio. Al contrario de lo que ocurre en los entornos tradicionales donde se espera de los estudiantes respuestas ya definidas, y un único camino para aprenderlas, en el sistema creado durante las sesiones coexisten varios resultados posibles y diferentes formas de llegar a ellos (Baek, 2009; MacMath, Roberts, Wallace y Chi, 2010). Por otra parte, el aprendizaje que los estudiantes realizan en el entorno simulado del videojuego causó un cambio sustancial, trasformando el espacio del problema y por lo tanto también su solución. Jugando con los SIM los problemas surgían en el mundo virtual y representaban situaciones de la vida cotidiana donde las decisiones tienen consecuencias inmediatas y funcionales, a diferencia de lo que ocurre en la enseñanza formal donde los resultados del aprendizaje son poco significativos para el aprendiz.

Los resultados obtenidos y su análisis a partir de la teoría de la actividad, evidencia la necesidad de construir nuevas herramientas conceptuales que tengan en cuenta el carácter dinámico de los procesos de aprendizaje, la interacción entre los diferentes elementos que determinan el cambio, y la trasformación que se genera por las contradicciones entre sistemas. Los resultados de este trabajo han revelado a la teoría de la actividad y su conceptualización diagramática y expansiva, como una herramienta potente para analizar el proceso de cambio cuando se incorpora un nuevo instrumento tecnológico como mediador en el aprendizaje. 


\section{Agradecimientos}

Este trabajo forma parte de un proyecto de investigación financiado por el Plan Nacional de I+D+i EDU2009-07075 (subprograma EDUC) (2009-2012). Agradecemos a los estudiantes del IES Manuel de Falla de Madrid (España) y a la profesora Rosa Piriz, su participación en el proyecto ha permitido llevar a cabo este trabajo.

\section{Referencias}

Abbott, C. (2007). E-inclusion: Learning Difficulties and Digital Techonologies. London: Kings College.

Back, Y, (2009). Digital Simulation in Teaching and Learning. En D. Gibson y Y. K. Baek (Eds.), Digital Simulations for Improving Education: Learning Through Artificial Teaching Environments (pp. 25-51). New York: Information Science Reference.

Blin, F., y Munro, M., (2008). Why hasn't technology disrupted academic's teaching practice?. Computers and Education, 50, 475-490. DOI:10.1016/j.compedu.2007.09.017

Cross, R (2010). Language Teaching as Sociocultural Activity: Rethinking Language Teacher Practice. The Modern Language Journal, 94(3), DOI:10.1111/j.15404781.2010.01058.x

Daniels, H. (2004). Cultural historical activity theory and professional learning. International Journal of Disability, Development and Education, 51(2), 185-200. DOI: $10.1080 / 10349120410001687391$

Engeström, Y. (2001). Expansive Learning at Work: toward an activity-theoretical reconceptualitation, Journal of Education and Work, 14(1), 133-156. DOI: $10.1080 / 13639080020028747$

Engeström,Y. (2008). From teams to knots: Activity-Theoretical Studies of Collaboration and Learning at Work. Cambridge: Cambridge University Press. DOI: 10.4018/jmbl.2009040101

Engeström,Y. (2009). Wildfire activities: New patterns of mobility and learning. International Journal of Mobile and Blended Learning, 1(2), 1-18.

Engeström,Y. (2014). Learning by Expanding. Cambridge: Cambridge University Press. 
Engeström, Y. y Sannino, A. (2010). Studies of expansive learning: Foundations, findings and future challenges. Educational Research Review, 5, 1-24. DOI:10.1016/j.edurev.2009.12.002

Gee, J.P. (2007). What Video Games Have to Teach Us About Learning and Literacy. Second Edition: Revised and Updated Edition [Paperback]. New York: Palgrave Macmillan.

Fujioka, M. (2014). L2 student-U.S. professor interactions through disciplinary writing assignments: An activity theory perspective. Journal of Second Language Writing, 25, 40-58. DOI:10.1016/j.jslw.2014.05.004

Hamera, J. (2011). Performance ethnography. En N. K. Denzin y Y. S. Lincoln (Eds.): The SAGE Handbook of Qualitative Research, 4th ed., 317-330. Thousand Oaks, Calif. London: Sage Publications.

Heemskerk, I,, Volman, M., Dam, G., y Admiraal, W. (2011). Social scripts in educational technology and inclusiveness in classroom practice. Teachers and Teaching: Theory and Practice, 17(1), 35-50. DOI: 10.1080/13540602.2011.538495

Janneke M., Frambach , J., Driessen., E., van der Vleuten, C. (2014). Using activity theory to study cultural complexity in medical education. Perspectives on Medical Education. 3, DOI: $10.1007 / \mathrm{s} 40037-014-0114-3$

Joy, R y Murphy, E. (2012). The Inclusion of Children with Special Educational Needs in an Intensive French as a Second-Language Program: From Theory to Practice. Canadian Journal of Education, 35(1), 102-119.

Juffs, A y Friedline, B. (2014). Sociocultural influences on the use of a web-based tool for learning English vocabulary. System, 42(2), 48-59. DOI: 10.1016/j.system.2013.10.015

Lacasa, P., Méndez, L., y Martínez, R. (2008). Developing new literacies using commercial videogames as educational tools. Linguistics y Education, 19(2), 85-106. DOI: 10.1016/j.linged.2008.02.001

Larripa, M., y Erausquin, C. (2010). Schooling practices and autism spectrum disorders: tools and challenges for the construction of inclusive school settings. A study from C.H.A.T. (cultural-historical activity theory) framework developed by Engeström. Anuario de Investigaciones, 17.

Leontiev, A. (1978). Activity, consciousness, and personality. Englewood Cliffs: PrenticeHall.

MacMath, S., Roberts, J., Wallace, J., y Chi, X. (2010). Curriculum integration and at-risk 
students: A Canadian case study examining student learning and motivation. British Journal of Special Education, 37, 87-94. DOI:10.1111/j.1467-8578.2009.00454.x

Méndez, L., Lacasa, P., y García-Pernía, M. (2013). Digital communities and videogames as educational tools in participatory culture. International Journal Social Media and Interactive Learning Environments, 1(2), 145-163. DOI: 10.1504/IJSMILE.2013.053602.

Monjelat, N., y Méndez, L. (2012). Problem solving Processes and Videogames: The Sim City Case, Electronic Journal of Research in Educational Psychology, 10(3), 14931522 .

Oswald, M. (2014). Positioning the individual Teacher in school-based learning for inclusion. Teaching and Teacher Education, 37, 1-10. http://dx.doi.org/10.1016/j.tate.2013.08.002

Patchen, T y Smithenry, D. (2014). Diversifying instruction and shifting authority: A cultural historical activity theory (CHAT) analysis of classroom participant structures. Journal of Research in Science Teaching, 51(5), 606-634. DOI: 10.1002/tea.21140

Phama, T y Renshawa, P. (2015). Formative assessment in Confucian heritage culture classrooms: activity theory analysis of tensions, contradictions and hybrid practices. Assessment $y$ Evaluation in Higher Education, 40(1), 45-59 DOI:10.1080/02602938.2014.886325

Säljö, R. (2010). Digital tools and challenges to institutional traditions of learning: technologies, social memory and the performative nature of learning. Journal of Computer Assisted Learning, 26(1), 53-64. DOI:10.1111/j.1365-2729.2009.00341.x

Swain,M., Xinnear, P., y Steinman, L. (2011). Sociocultural theory in second language education: An introduction through Narratives. Bristol, UK: Multilingual Matters.

Watson, W. R., Mong, C. J., y Harris, C. A. (2011). A case study of the in-class use of a video game for teaching high school history. Computers y Education, 56(2), 466-474. DOI: 10.1016/j.compedu.2010-09-007.

Yamagata-Lynch, L y Haudenschild, M. (2009). Using activity systems analysis to identify inner contradictions in teacher professional development. Teaching and Teacher Education, 25, 507-517. DOI:10.1016/j.tate.2008.09.014

Yamagata-Lynch, L, (2010). Activity Systems Analysis Methods. Understanding Complex Learning Environments. New York: Springer.

Yin, R. (2011). Applications of Case Study Research. California: SAGE Publications. 
Laura Méndez et al.

[Página en blanco por razones de paginación] 